\title{
Optimization and validation of PD-L1 immunohistochemistry staining protocols using the antibody clone $28-8$ on different staining platforms
}

\author{
Christina Koppel ${ }^{1} \cdot$ Helena Schwellenbach ${ }^{1} \cdot$ Dirk Zielinski $^{1} \cdot$ Sina Eckstein $^{2} \cdot$ Mercedes Martin-Ortega $^{2}$. \\ Corrado D'Arrigo $^{3} \cdot$ Hans-Ulrich Schildhaus ${ }^{2} \cdot$ Josef Rüschoff $^{1,4} \cdot$ Bharat Jasani $^{1}$
}

Received: 17 November 2017 / Revised: 5 April 2018 / Accepted: 8 April 2018 / Published online: 26 June 2018

(c) United States \& Canadian Academy of Pathology 2018

\begin{abstract}
Several immunohistochemistry (IHC) assays have been developed to assess tumor programmed death-ligand 1 (PD-L1) expression levels in patients who are candidates for programmed death-1 (PD-1)/PD-L1 inhibitor therapy. The PD-L1 IHC 28-8 pharmDx kit is FDA-approved as a complementary diagnostic and CE-marked as an in vitro diagnostic device for nivolumab therapy in melanoma and specific lung cancer subtypes (and for squamous cell carcinoma of the head and neck/ urothelial carcinoma in Europe only). Kit availability is limited outside the United States, and its use requires the Dako Autostainer Link 48 platform, which is unavailable in many laboratories. Validated laboratory-developed tests based on 28-8 concentrated antibody outside the kit are needed. This study compared the results from PD-L1 expression level analysis across four immunohistochemistry platforms (Dako Autostainer Link 48, Dako Omnis, Leica Bond-III, and Ventana BenchMark ULTRA) with the 28-8 pharmDx kit in lung cancer (multiple histologies), melanoma, and head and neck cancer (multiple histologies). Samples were prepared per protocol for each platform and stained using PD-L1 IHC 28-8 pharmDx kit on Dako Autostainer Link 48, and per protocol for each platform. The control samples (tonsil and placenta tissue; cell lines with prespecified PD-L1 expression levels) were tested to evaluate the specificity and the sensitivity of test assays. An agreement level of 0.90 with the pharmDx kit was set for each platform. Inter- and intra-assay reliability were assessed. Evaluable samples were lung cancer $=29$; melanoma $=31$; head and neck cancer $=30$. Mean agreement was calculated for PD-L1 expression levels of $\geq 1 \%, \geq 5 \%, \geq 10 \%$, and $\geq 50 \%$. Mean overall agreement for all indications was $0.87-0.99$. Interand intra-assay of scoring/classification repeatability was $100 \%$. Analysis of PD-L1 expression levels using laboratorydeveloped immunohistochemistry assays with 28-8 antibody may be permissible if the platform is validated using reference samples with defined expression levels.
\end{abstract}

These authors contributed equally: Christina Koppel, Helena Schwellenbach.

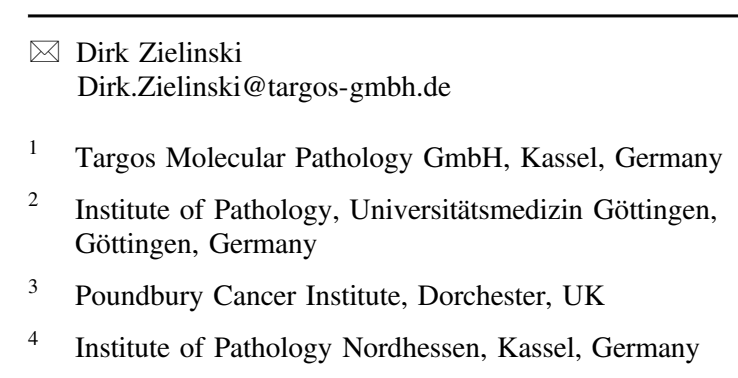

\begin{tabular}{ll}
\hline 凶 & Dirk Zielinski \\
& Dirk.Zielinski@targos-gmbh.de \\
1 & Targos Molecular Pathology GmbH, Kassel, Germany \\
2 & $\begin{array}{l}\text { Institute of Pathology, Universitätsmedizin Göttingen, } \\
\text { Göttingen, Germany }\end{array}$ \\
3 & Poundbury Cancer Institute, Dorchester, UK \\
4 & Institute of Pathology Nordhessen, Kassel, Germany
\end{tabular}

\section{Introduction}

Programmed cell death-1 (PD-1) receptor and its ligands, programmed cell death-ligands 1 and 2 (PD-L1, PD-L2), are important checkpoint regulators in immune cells [1-3]. PDL1 and PD-L2 are frequently expressed in many cancer types, including non-small cell lung cancer, melanoma, and squamous cell carcinoma of the head and neck [4-6]. Binding of these ligands to PD- 1 has been shown to impair the activity of cytotoxic $\mathrm{T}$ cells $[1,2,4,7]$. This phenomenon enables tumor cells to evade the body's natural immune defense mechanism, thereby allowing cancers to progress. Studies have shown that blockade of PD-1 restores the immune function of T cells [8]. Assessment of PD-L1 expression using validated diagnostic antibodies may therefore be useful in predicting the response to anti-PD-1/PD-L1 treatment. 
Nivolumab was the first PD-1 immune checkpoint inhibitor to be approved worldwide; it was licensed in Japan in 2014 for the treatment of unresectable malignant melanoma [9]. Since then, nivolumab and other PD-1/PD-L1 inhibitors, including pembrolizumab and atezolizumab, have been approved for use in patients with previously treated locally advanced and/or metastatic non-small cell lung cancer [10-15]. Pembrolizumab is also approved for previously untreated metastatic non-small cell lung cancer. These PD1/PD-L1 inhibitors, along with the PD-L1 inhibitor durvalumab, have also been approved for the treatment of various other tumor types [10-16]. In metastatic non-small cell lung cancer, treatment with pembrolizumab currently requires assessment of tumor PD-L1 expression, either with the companion diagnostic the PD-L1 IHC 22C3 pharmDx kit (Agilent Technologies, Santa Clara, CA) (U.S. requirement) [11] or with any other validated immunohistochemistry (IHC) assay [14]. For the treatment of non-small cell lung cancer with pembrolizumab, PD-L1 levels must be $\geq 50 \%$ in treatment-naive patients and $\geq 1 \%$ in previously treated patients $[11,14]$. There is no companion diagnostic required for treatment with nivolumab, although a U.S. Food and Drug Administration (FDA)-approved complementary diagnostic is available for non-squamous non-small cell lung cancer and melanoma, the PD-L1 IHC 28-8 pharmDx kit (Agilent Technologies, Santa Clara, CA) [10, 17, 18]. This diagnostic assay is also available in Europe as a CEmarked in vitro diagnostic device for the same lung and melanoma indications, and for patients with squamous cell carcinoma of the head and neck or urothelial carcinoma who may benefit from nivolumab $[18,19]$.

Extensive research has examined the value of tumor PDL1 expression levels, both in assessing the disease prognosis and in predicting the response to anti-PD-1/PD-L1 immunotherapies. Higher PD-L1 expression levels are associated with poor prognosis, independently of treatment, in some tumor types, including non-small cell lung cancer [20-23]. However, other studies have found no association with prognosis in PD-L1-expressing non-small cell lung cancer or melanoma [6, 23, 24]. The association between PD-L1 expression levels and treatment response may be related to tumor histology (e.g., squamous cell vs. adenocarcinoma) [21, 25-30]. The value of PD-L1 expression as a predictor of response to anti-PD-1/PD-L1 treatments may also differ depending on whether the patients with advanced or metastatic disease receive these treatments as first-line therapy, or after previous therapy. In first-line treatment of patients with advanced squamous and non-squamous non-small cell lung cancer with high PD-L1 expression levels ( $\geq 50 \%$ of tumor cells), treatment with the PD-1 inhibitor pembrolizumab was associated with longer progression-free and overall survival, compared with chemotherapy [31]. In contrast, another study with lower prespecified PD-L1 expression levels ( $\geq 5 \%$ of tumor cells) found that PD-1 inhibitor therapy showed similar outcomes to chemotherapy [32].

Several factors contribute to difficulties in the assessment of PD-L1 expression. PD-L1 induction in tumors results from either oncogenic signaling, leading to constitutive widespread expression, or in response to IFN- $\gamma$ release by effector $\mathrm{T}$ cells during their immune response to the tumor, leading to variable expression of inducible PD-L1 [33, 34]. Prior therapy may therefore alter the expression of inducible PD-L1, as well as other biomarkers that form part of the dynamic immune landscape of the tumor and tumor microenvironment and interact with PD-L1 [26, 35-37]. Levels of constitutive versus inducible PD-L1 may vary across tumor types, with predominant expression of IFN- $\gamma$-induced PD-L1 in melanoma, and a lower level of constitutive PD-L1 in melanoma compared with lung tumors [37]. The spatial and temporal heterogeneity of induced PD-L1 also means that the location and timing of the biopsy and type of sample (for example, biopsy vs. surgical resection) can influence PD-L1 expression levels $[26,38]$.

At the epitope level, there may also be difficulties in assessing PD-L1 expression as a biomarker of response to anti-PD-1/PD-L1 treatments. Currently, only PD-L1 in the cell membrane, rather than intracellular protein or mRNA, is assessed [23]. However, in cells with strong cytoplasmic staining, it can be difficult to determine the extent of membranous staining [39]. The structure of PD-L1, with two small linear hydrophilic binding regions, restricts the number of sites available for antibody binding [23, 26, 40], which makes detection using typical immunohistochemistry methods on formalin-fixed, paraffin-embedded specimens difficult $[23,26]$. This means that immunohistochemistry antibodies typically bind PD-L1 at sites different from the therapeutic antibodies [26]. Linear epitope mapping of anti-PD-L1 antibodies, including 28-8, has suggested that potential conformational epitopes of PD-L1 targeted by the anti-PD-L1 antibody may affect the staining patterns [41].

The PD-L1 IHC 28-8 pharmDx kit is a validated [4245], automated immunohistochemistry assay using Monoclonal Rabbit Anti-PD-L1, Clone 28-8 (Agilent Technologies, Santa Clara, CA) in combination with the Dako Autostainer Link 48 platform. Outside the United States, pathologists have expressed an interest in validated staining protocols [46] using the rabbit monoclonal antihuman PDL1 antibody (clone 28-8) on common staining platforms. These validated protocols may be useful when the PD-L1 IHC 28-8 pharmDx kit or Dako Autostainer Link 48 platform are difficult to access. To this end, we have developed and validated immunohistochemistry staining protocols using the anti-PD-L1 28-8 antibody purchased from Abcam (Cambridge, UK) [47] on four major automated staining 
platforms: Dako Autostainer Link 48 (Agilent Technologies, Santa Clara, CA) [48]; Dako Omnis (Agilent Technologies, Santa Clara, CA) [49]; Leica Bond-III (Leica Biosystems, Wetzlar, Germany) [50]; and Ventana BenchMark ULTRA (Ventana Medical Systems, Roche Diagnostics, Tucson, AZ [software version 12.3]) [51]. Results from each of these four staining protocols were compared with those from the PD-L1 IHC 28-8 pharmDx kit on the Dako Autostainer Link 48 applied to PD-L1-expressing and non-PD-L1-expressing samples of lung cancer (various histologies), melanoma, and head and neck cancer (various histologies), together with batch tissue controls (tonsil and placenta) and reference cell lines with defined levels of PDL1 expression (\#HD787, Horizon Discovery Group, Cambridge, UK) [52]. This study was designed to examine whether PD-L1 expression levels assessed using four widely available platforms produced comparable results to the FDA-approved and CE-marked IHC 28-8 pharmDx kit.

\section{Materials and methods}

\section{Sample preparation}

Formalin-fixed, paraffin-embedded sample specimens from surgical resections were obtained from the archive of the Institute of Pathology Nordhessen (Kassel, Germany) following ethical approval by the Landesärztekammer Hessen. Additional samples were purchased from a commercial biobanking service (Cureline, Inc., San Francisco, CA). Evaluable tumor samples included human lung cancer (total $n=29: 3$ squamous cell carcinoma, 7 adenocarcinoma, 16 indeterminate, 1 sarcomatoid, 2 large-cell carcinoma); melanoma $(n=31)$; head and neck cancer (total $n=30$ : 21 squamous cell carcinoma, 9 adenocarcinoma). Individual patient informed consent had been obtained for all samples. All samples were stained once with the PD-L1 IHC 28-8 pharmDx kit to determine the reference PD-L1 expression levels.

A comparison of assay results on different staining platforms was performed on tissue microarrays prepared from one $1.5 \mathrm{~mm}$ diameter core per donor block. The tissue microarrays were prepared and analyzed at Targos Molecular Pathology GmbH, Kassel, Germany, or the Institute of Pathology, Universitätsmedizin Göttingen, Göttingen, Germany (for Dako Omnis platform). From each tissue microarray, serial sections of $4-5 \mu \mathrm{m}$ were cut, mounted onto Fisherbrand Superfrost Plus charged slides, and placed in an oven at $58^{\circ} \mathrm{C}\left( \pm 2{ }^{\circ} \mathrm{C}\right)$ for $1 \mathrm{~h}$ (except for samples prepared for the Dako Omnis platform, where the slides were placed in the oven at $65{ }^{\circ} \mathrm{C}$ for $20 \mathrm{~min}$, according to local protocol). The sections were deparaffinized in xylene, then rehydrated in a standard series of descending alcohol immersions and stained with the immunohistochemistry protocols described in Table 1. After each run, on all platforms, slides were dehydrated in ascending alcohol, ending in xylene, and coverslipped using a non-aqueous mounting medium and standard glass coverslips. Tissue microarray cores were excluded from the analysis if they were washed off, did not contain tumor, or the sample was not evaluable for other reasons.

\section{Staining of control samples}

Tonsil epithelium and placental syncytiotrophoblast were prepared as above and stained as batch control samples, one with Abcam anti-PD-L1 28-8 antibody and a second specimen with negative control reagent (Table 1). A PD-L1 standard microarray (\#HD787, Horizon Discovery Group, Cambridge, UK) [52] comprising cell lines with controlled PD-L1 expression (negative $[-]$, low positive $[+]$, intermediate positive $[++]$, and strong positive $[+++]$ ) provided an additional control. The samples were pre-analyzed for PD-L1 expression using the PD-L1 IHC 28-8 pharmDx kit to verify the immunohistochemistry performance. PD-L1 expression levels were then determined by immunohistochemistry using the Abcam anti-PD-L1 28-8 antibody on each of the four platforms detailed below. Validation was performed according to all relevant College of American Pathologists/Clinical Laboratory Improvement Amendments criteria for immunohistochemistry assays.

\section{Dako PD-L1 28-8 pharmDx kit}

The immunohistochemistry staining protocol using the PDL1 IHC 28-8 pharmDx kit was performed as previously described [43] and as specified in the pharmDx kit label [18]. Slides were processed on the Autostainer Link 48 (Agilent Technologies, Santa Clara, CA) alongside control slides provided with the kit, using an automated staining protocol validated for the PD-L1 immunohistochemistry assay, and prepared using EnVision FLEX Target Retrieval Solution, Low pH (Dako, Glostrup, Denmark).

\section{Assay establishment and validation procedure}

For each of the staining platforms, protocols were established starting as closely similar as possible to the original PD-L1 28-8 pharmDx kit protocol. For the application of the 28-8 antibody on the Dako Autostainer Link 48, this included the use of all reagents (Detection Kit, Wash Buffer, Antibody Diluent) from Dako and application of the same incubation conditions as in the pharmDx assay (Table 1). The concentration of the primary antibody was then adjusted by titration series $(23.84-2.98 \mu \mathrm{g} / \mathrm{mL})$. The same procedure was used to adapt the protocol for the Dako 


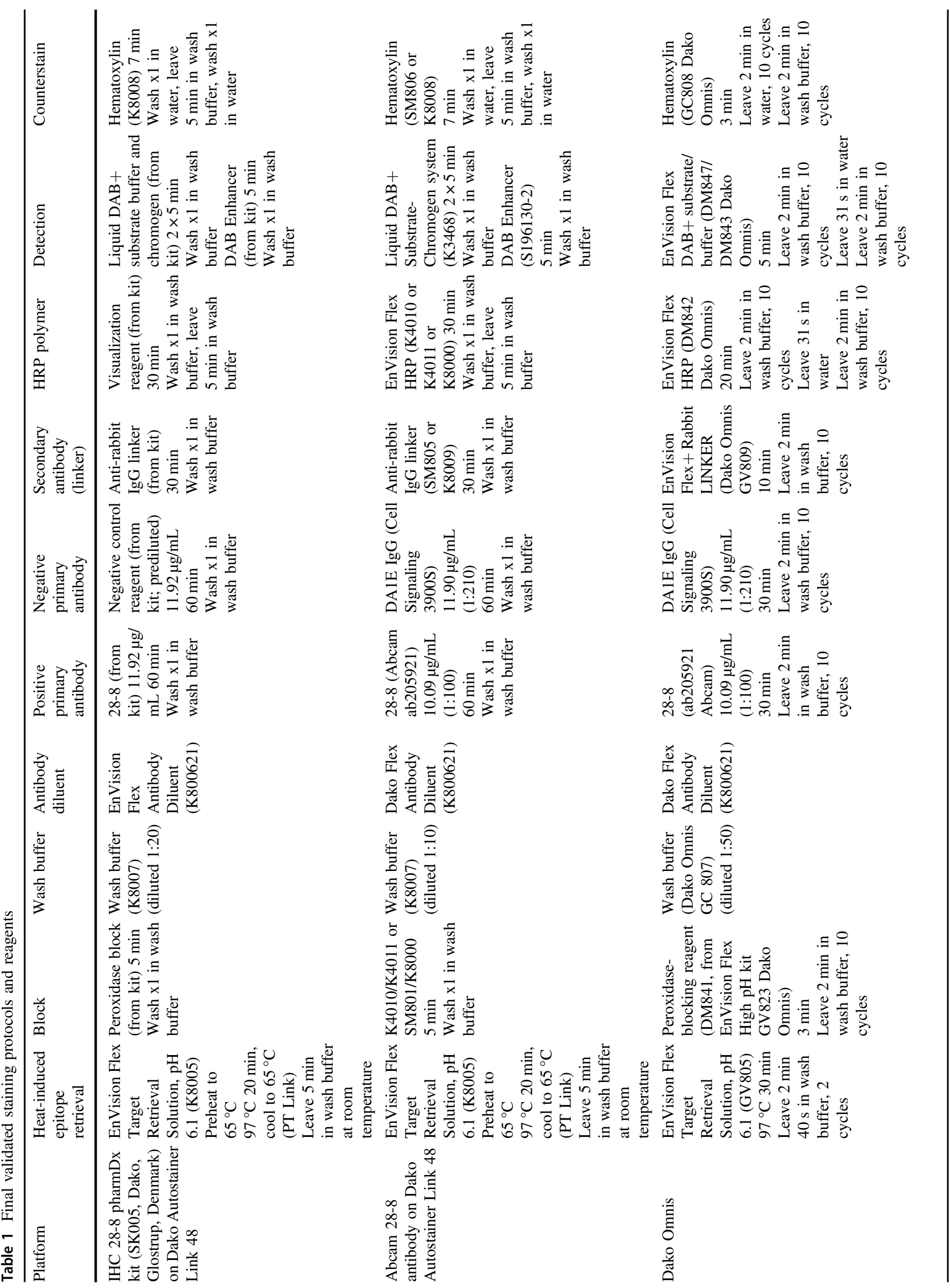




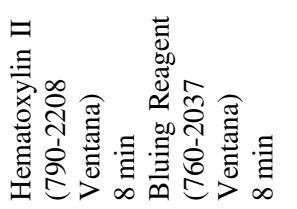

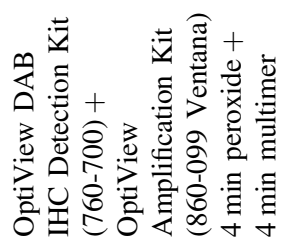

裵

总

总高塄

番

글

密 홍

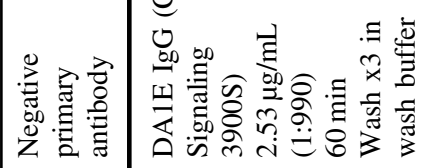

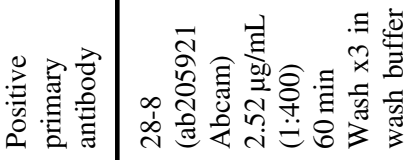

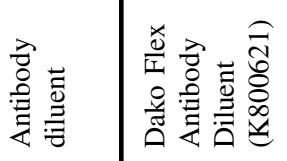

离

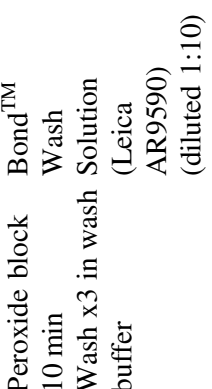

它

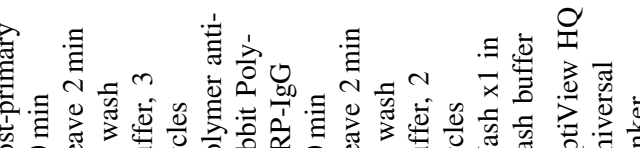

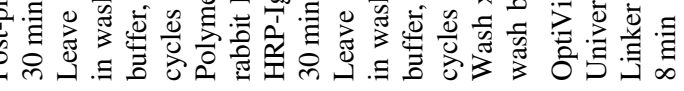

3

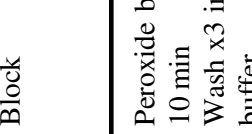

ఫัँ

西

迹 $\frac{0}{2} \cdot \frac{0}{2}$

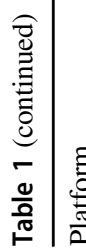

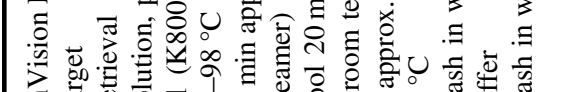

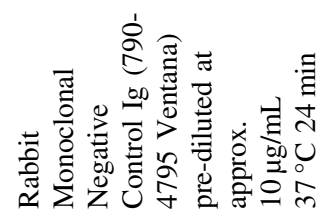

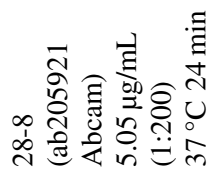

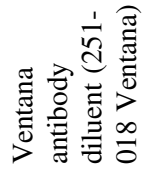

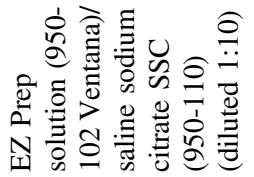

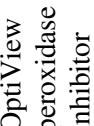

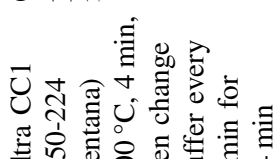

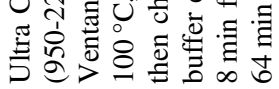

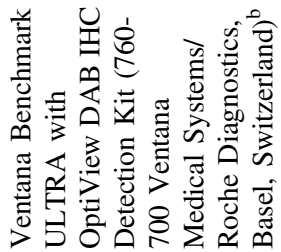

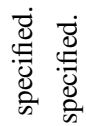

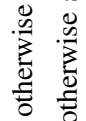

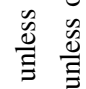

过

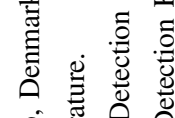

言芯卷

웜 $\cong$

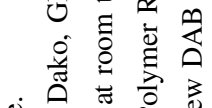

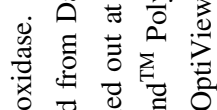

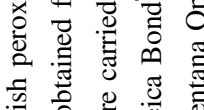

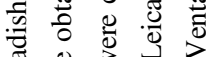

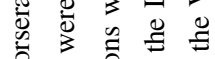

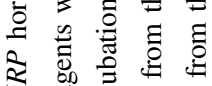

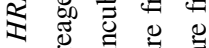

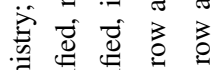

एँ

品

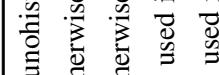

言焉

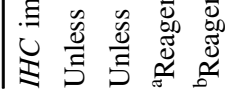


Omnis platform, with antibody concentrations ranging from 11.92 to $2.98 \mu \mathrm{g} / \mathrm{mL}$ and Dako FLEX and FLEX + detection systems being tested. Most importantly, the antigen retrieval conditions were not changed from the original pharmDx protocol. For the remaining staining platforms, the pretreatment buffers and detection systems selected differed from the Dako reagents. Originally, similar pretreatment conditions were used on Leica Bond-III; however, neither Leica ER2 nor ER1 solutions yielded satisfactory results, and were replaced by external heat-induced epitope retrieval using EnVision FLEX Target Retrieval Solution, Low pH in a steamer (Table 1). Tested primary antibody concentrations on the Leica Bond-III ranged from 47.68 to $2.52 \mu \mathrm{g} / \mathrm{mL}$ and consisted of double incubation of the antibody and a signal amplification step using FLEX+Rabbit Linker antibody from Dako (final protocol is specified in Table 1). While establishing a protocol for the Ventana BenchMark ULTRA, we investigated a range of incubation times for $\mathrm{CC} 1$ pretreatment (8-64 min), primary antibody concentrations $(15.89-5.96 \mu \mathrm{g} / \mathrm{mL})$, and incubation times (30-60 min), respectively. The OptiView DAB detection system including an additional signal amplification step was required for the Ventana platform as a substitute for the less sensitive, but more commonly used, UltraView system.

All final protocols were confirmed on full sections of formalin-fixed, paraffin-embedded tonsil and placenta specimens, as well as on the selected lung cancer samples and the defined cell-line control slides (Horizon). Validation of the final protocols listed, specified in Table 1, was conducted, as described below.

\section{Repeatability}

Inter- and intra-assay repeatability was determined by repeated staining of the selected lung cancer samples (low, medium, and high levels of PD-L1 expression) in three independently performed assay runs, and by staining the three replicates of those specimens in the same assay run, respectively.

\section{Stability and matrix effect}

Antigen stability in slide-mounted sections was checked over a period of 6 weeks in total. The effect of various fixatives (10\% neutral buffered formalin, $10 \%$ non-buffered formalin, AFA, Pen-Fix, Prefer, and Z-5) and fixation times were tested at room temperature using tissue microarrays prepared from specimens derived from the same tumor, but fixed differently.

\section{Sample evaluation and statistical analysis}

Semi-quantitative determination of PD-L1 expression levels using bright-field microscopy was performed by senior pathologists experienced and trained in PD-L1 immunohistochemistry evaluation and involved either in clinical trials with PD-L1 as a biomarker (BJ, JR) or in a worldwide training program for $28-8$ as a predictive biomarker (CD'A, H-US). Specimens in each tumor category were read by a single pathologist. Three samples (one exhibiting high, one medium, and one low biomarker concentration) were tested in a single assay run. All samples were blinded and analyzed singly. Raw data were documented on customized forms, $2 \times 2$ contingency tables were generated, and positive, negative, and overall agreement were calculated using Microsoft Excel 2007.

\section{Interpretation}

Staining interpretation (PD-L1 on tumor cells) was performed on the basis of the official interpretation manual of Dako for Monoclonal Rabbit Anti-PD-L1, Clone 28-8 pharmDx kit (Agilent Technologies, Santa Clara, CA). The percentage of tumor cells with PD-L1-membranous staining, regardless of intensity, was determined. Non-malignant and immune cells, cytoplasmic staining, necrotic cells, and debris were excluded. A sample was considered evaluable if at least 100 viable tumor cells were present.

PD-L1-positive tumor cells were quantified by evaluating the ratio of area proportion of stained to unstained tumor cells according to custom scoring criteria of the assay [17, 18]. Prespecified PD-L1 expression levels of $\geq 1 \%, \geq 5 \%, \geq 10 \%$, and $\geq 50 \%$ tumor cell staining were used for final classification of all samples into positive or negative categories for each threshold level. This scoring algorithm was applied to all platforms and the scores obtained for each platform were compared with the respective scores obtained using the PDL1 IHC 28-8 pharmDx kit as the reference assay.

An agreement level of 0.90 , based on the final PD-L1positive/negative classifications with respect to the expression thresholds, was used to decide whether each platform provided acceptable results in comparison with the PD-L1 IHC 28-8 pharmDx kit.

\section{Results}

All staining protocols used resulted in the characteristic tumor cell pattern of membranous staining with an accompanying cytoplasmic component. The membranous staining obtained with the Leica Bond-III and Ventana BenchMark ULTRA platforms was less clearly defined, compared with the Dako platforms (Autostainer Link 48 and Omnis). The observed percentage, intensity, and distribution of the stained cells covered a wide dynamic range from negative $(0 \%)$ to strongly positive staining and from focal to homogenous staining of the tumor cells (Fig. 1), 


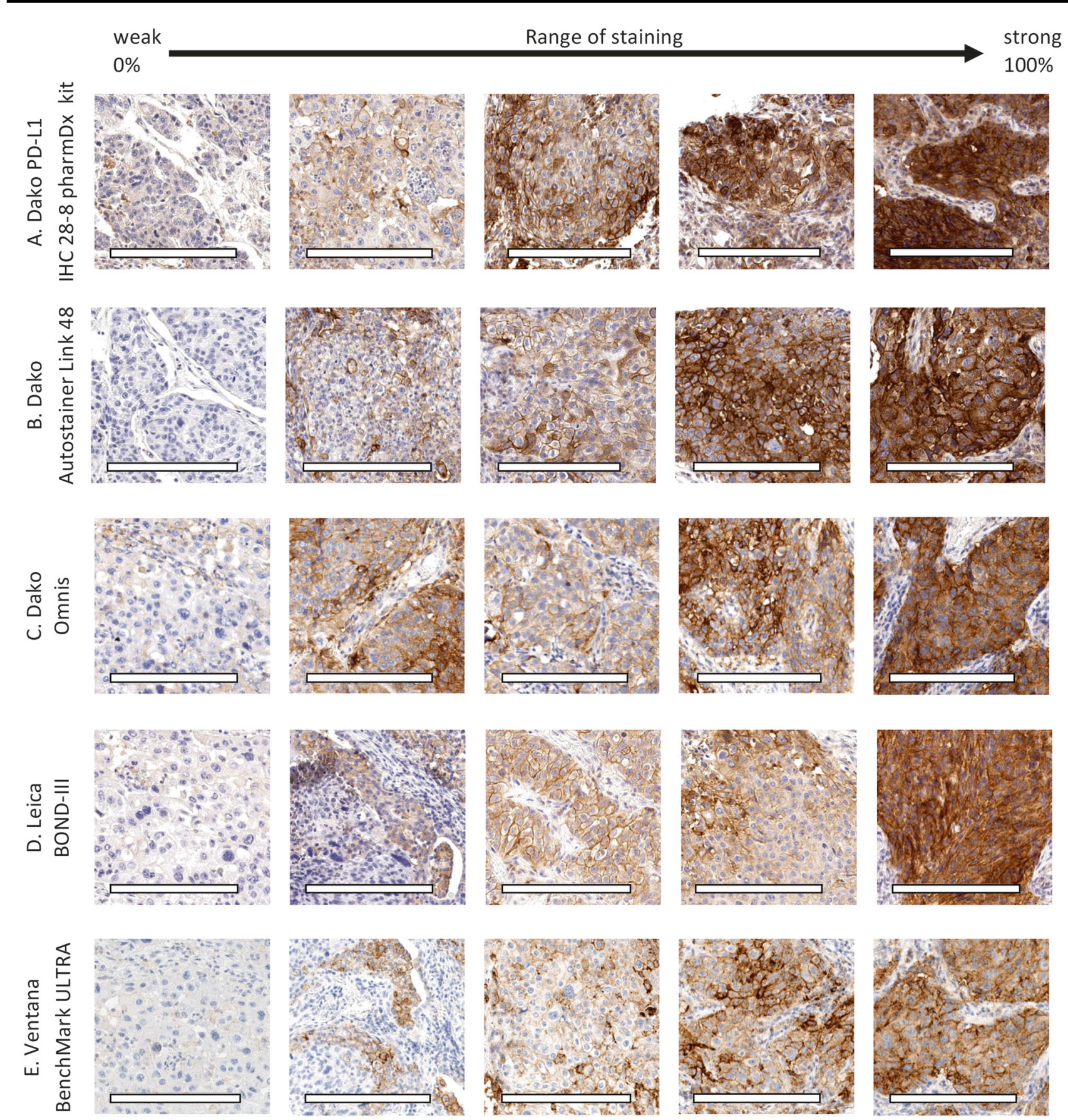

Fig. 1 PD-L1 immunohistochemistry staining in the lung cancer tumor microarray samples displaying different staining intensity (non-matched sections), using antibody clone 28-8 within the pharmDx kit and outside the kit different automated staining platforms. a Dako PD-L1

and was found to be comparable among the different platforms, compared with the PD-L1 IHC 28-8 pharmDx kit (Table 2).

\section{Repeatability}

For the three selected lung cancer samples, there was $100 \%$ inter- and intra-assay repeatability scoring/classification
IHC 28-8 pharmDx kit. b Dako Autostainer Link 48. c Dako Omnis. d Leica Bond-III. e Ventana BenchMark ULTRA (All images 20×, bar $=200 \mu \mathrm{m}$, Aperio Scanscope AT2)

(Table 3), with acceptable coefficient of variation in percentages of positively stained target cells.

\section{Specificity of immunohistochemistry staining across platforms}

All protocols adequately stained the tonsil epithelium (Fig. 2) and placental syncytiotrophoblast (Fig. 3), used as 


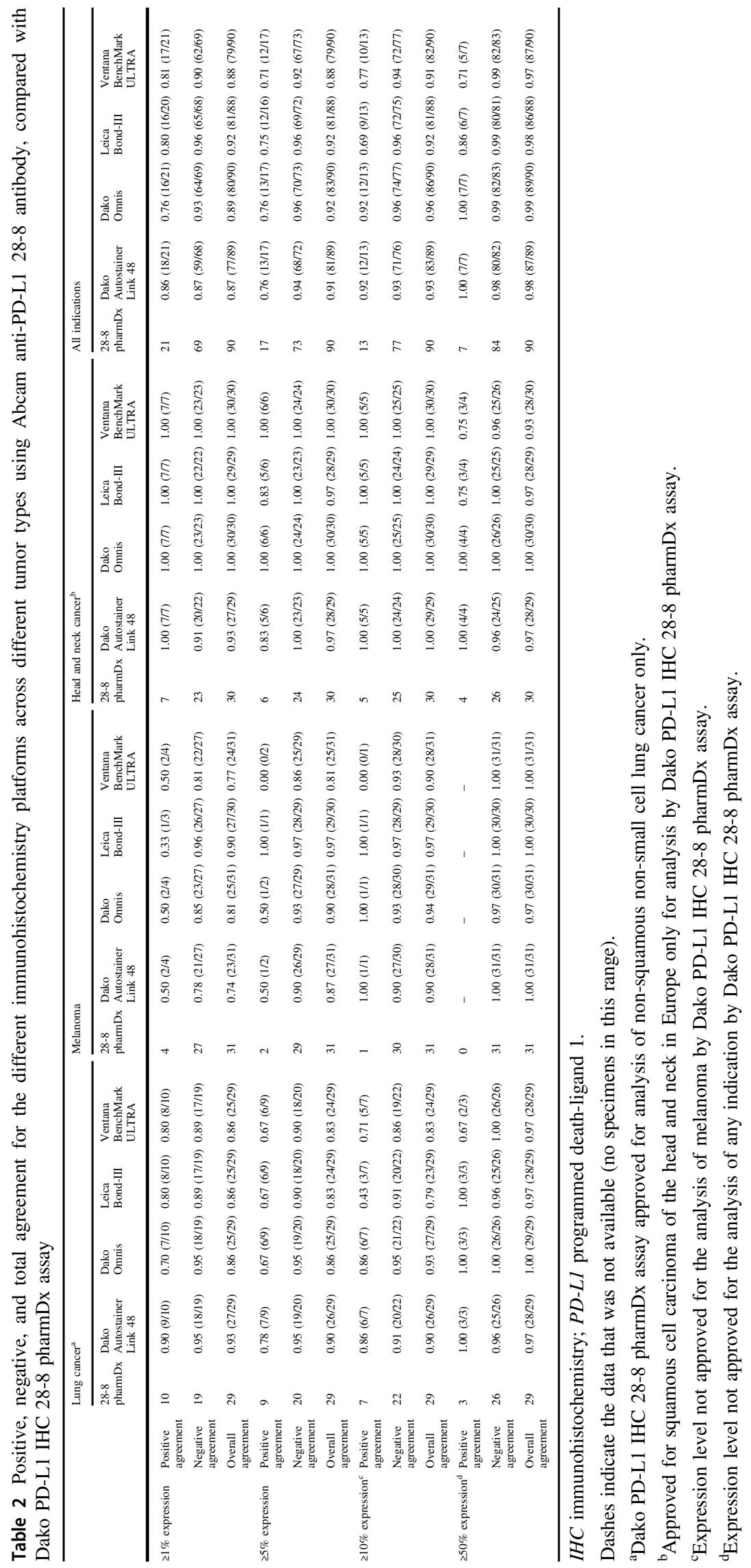




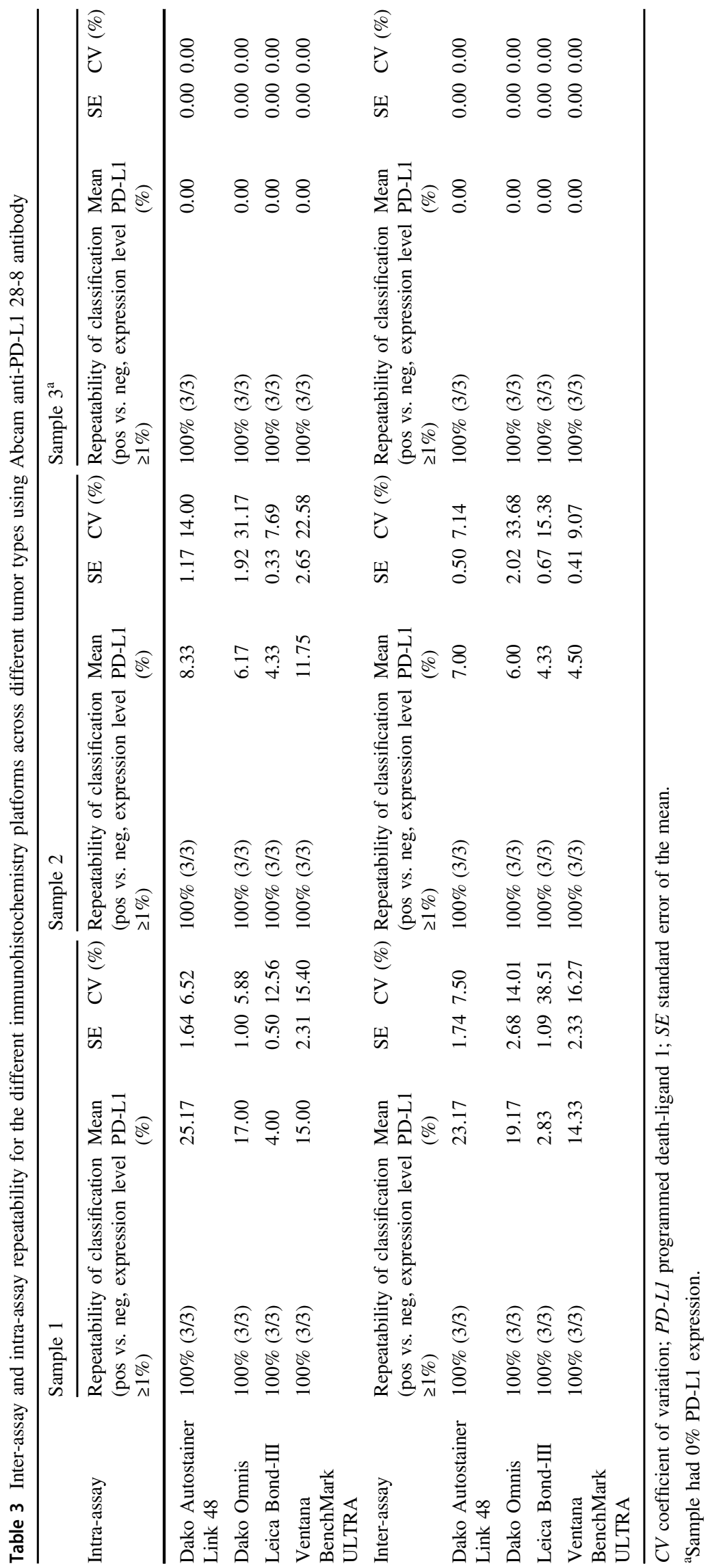



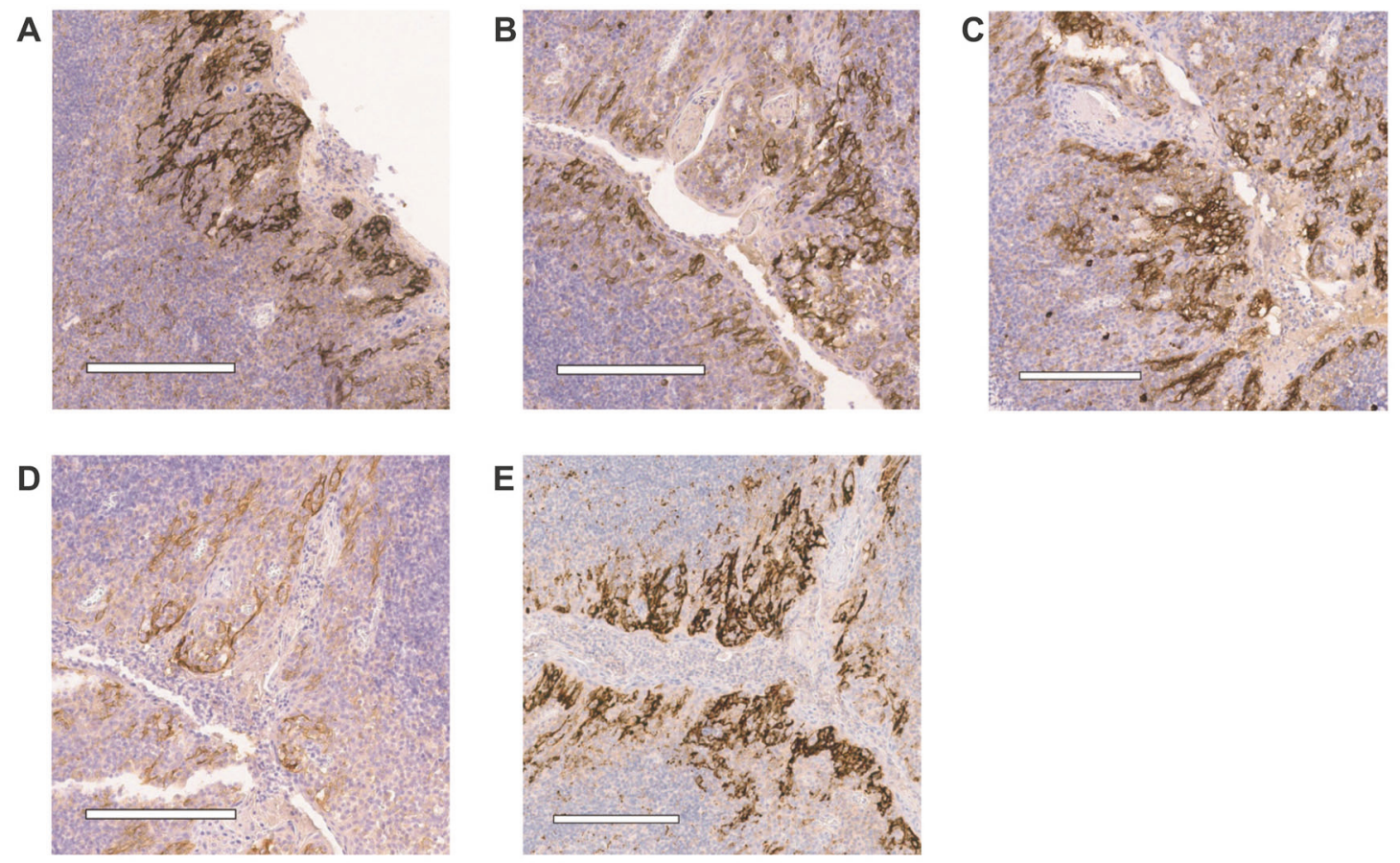

Fig. 2 PD-L1 immunohistochemistry staining in tonsil (positive control tissue) using clone 28-8 within the pharmDx kit and outside the kit on different automated staining platforms. a Dako PD-L1 IHC 28-8

batch positive controls. Thus, the expression of the target antigen could be detected in seven of the seven tonsil and six of the six placenta specimens. A tissue microarray comprising samples from all major human organs (created within Targos Molecular Pathology $\mathrm{GmbH}$ ) was also tested to verify the specificity of PD-L1 expression in human tissues. Using all immunohistochemistry staining platforms and the pharmDx kit, no physiological presence of PD-L1 expression could be detected in prostate, testis, ovary, fallopian tube, uterus, adrenal gland, colon, stomach, heart, cerebellum, cerebrum, or thyroid gland. Nuclear staining in distal tubules of the kidney was detected in four of the six samples. Liver specimens exhibited non-specific cytoplasmic grossly granular staining in four of the six cases, most likely representative of hemosiderin deposits. The majority of samples across all tumor types had $<1 \%$ PD-L1 expression, as determined by the PD-L1 IHC 28-8 pharmDx kit (lung cancer 19/29, melanoma 27/30, head and neck cancer 23/30). Depending on the expression level and the tumor indication, negative agreements ranged from 0.74 to 1.00 ; in most cases, the values for negative agreement were below the specified target value of 0.90 (Table 2).

\section{Sensitivity}

All protocols successfully stained a defined PD-L1 standard cell-line microarray, with cell lines negative (-), low positive $(+)$, intermediate positive $(++)$, and strong
pharmDx kit. b Dako Autostainer Link 48. c Dako Omnis. d Leica Bond-III. e Ventana BenchMark ULTRA (All images 11×, bar $=200$ $\mu \mathrm{m}$, Aperio Scanscope AT2)

positive $(+++)$ for PD-L1 expression. Figure 4 shows comparable levels of staining and percentages of PD-L1expressing cells per cell line [52].

\section{Stability}

Despite the observed variations in staining intensity and patterns after storage at room temperature, antigen stability over a 6-week period did not influence the final classification in these samples (PD-L1 expression levels above or below the prespecified expression levels). Compared with the original staining pattern in $10 \%$ neutral buffered formalin and non-buffered formalin, all non-formalin-based fixatives showed a marked, but not systematic, change in actual staining patterns in appearance, staining intensity, and number of affected cells (up to and including change to 0\% PD-L1 expression, i.e., false-negative staining) (data not shown).

\section{Discussion}

A systematic comparison of PD-L1 expression levels in lung cancer, melanoma, and head and neck cancer tissue microarrays assessed by four major automated staining platforms using the Abcam anti-PD-L1 28-8 antibody revealed adequate precision and concordance when compared with the recommended complementary diagnostic kit 

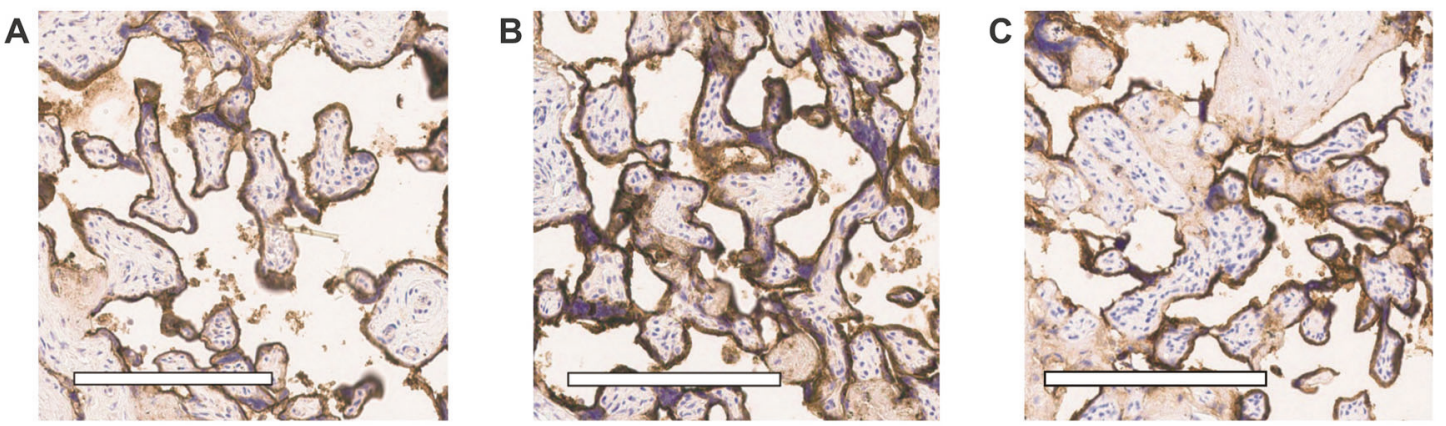

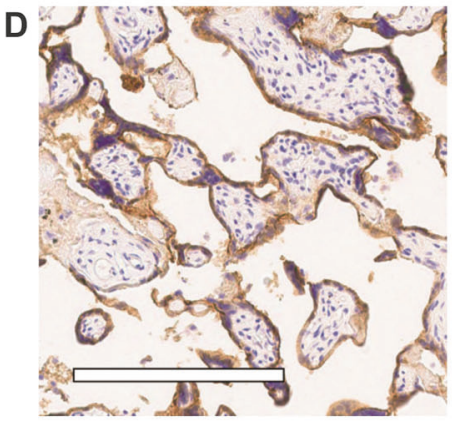

Fig. 3 PD-L1 immunohistochemistry staining in placenta (positive control tissue) using clone 28-8 within the pharmDx kit and outside the kit on different automated staining platforms. a Dako PD-L1 IHC 28-8
pharmDx kit. b Dako Autostainer Link 48. c Dako Omnis. d Leica Bond-III. e Ventana BenchMark ULTRA (All images 16×, bar $=200$ $\mu \mathrm{m}$, Aperio Scanscope AT2)

Positive control tonsil and placenta specimens were all found to have high PD-L1 expression levels, suggesting that they may not provide a reliable enough control sample for assessing the relative sensitivity of a given protocol setup. However, cell-line microarrays, which included a range of cell lines showing negative PD-L1 staining to strongly positive PD-L1 staining, provided a more effective control, allowing demonstration of high sensitivity and specificity for assays on all platforms. To validate future protocols, the use of reference tissue specimens and/or cell lines with defined expression levels covering the entire expected range is therefore strongly recommended, in addition to the tonsil and placenta tissue specimens being used, with tonsil as onslide or run-related batch controls.

The staining of tumor samples remained stable (in terms of maintaining categorization above or below prespecified expression levels) over a period of 6 weeks, although testing with different fixatives and fixation times revealed that non-formalin-containing fixatives resulted in marked changes in staining pattern and intensity, compared with the original staining pattern. The recommended fixatives thus include $10 \%$ neutral buffered formalin, as well as nonbuffered formalin, applied for 18-24 h. Based on our findings, non-formalin-containing fixatives should be avoided. Outside the current study, however, it has been observed with clinical samples that long-term storage of unstained slides at room temperature leads to a significant reduction of PD-L1 staining intensity. Therefore, the use of archival 


$\begin{array}{ccccc} & \text { Negative } & \text { Low positive } & \text { Intermediate positive } & \text { Strong positive } \\ & - & + & ++ & +++\end{array}$
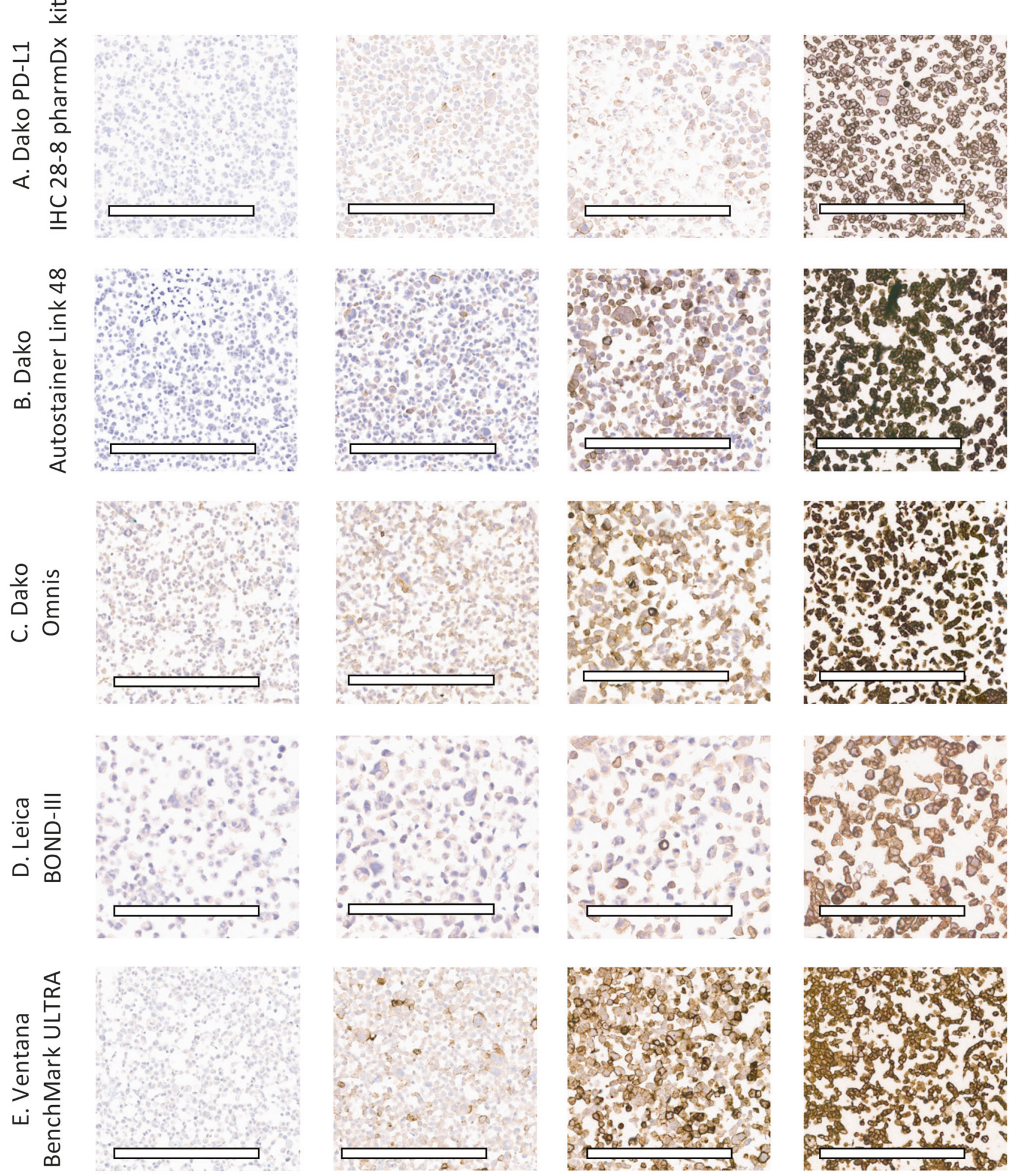

Fig. 4 PD-L1 immunohistochemistry staining of negative (-), low positive $(+)$, intermediate positive $(++)$, and strong positive $(+++)$ protein expressing cell lines. a Dako PD-L1 IHC 28-8 pharmDx kit. b Dako Autostainer Link 48. c Dako Omnis. d Leica Bond-III. e Ventana BenchMark ULTRA (All images 20x, bar $=200 \mu \mathrm{m}$, Aperio Scanscope AT2) 
sections that have been stored for longer than 3 months is not recommended.

The small cohort size and the increased likelihood of small tissue specimens such as tissue microarray cores detaching from slides (which reduced the number of evaluable samples) provided some technical limitations. Tissue microarrays were used in the current analysis for an assayto-assay comparison between staining protocols, and prepared as parallel sections in order to minimize variability. Consideration of intratumoral heterogeneity as a potential confounding factor was not within the scope of this study, though it should be considered as a concern when using tissue microarrays, as it could result in variable levels in PD-L1 expression, depending on the degree of heterogeneity [62-64]. It should be noted that the pharmDx kit has not been validated for tissue microarrays, and can result in lower levels of PD-L1 than found in clinical practice [65]. However, the current study found similar levels and patterns of PD-L1 expression in tissue microarrays across all laboratory-developed tests compared with the pharmDx kit. This supports the applicability of this study to clinical practice, notwithstanding the generally acknowledged limitations of tissue microarray-based analysis. Since cytological specimens may be more easily and less invasively obtained than histological specimens, the performance of assays in different sample types has been examined previously and found to be highly concordant using both the PD-L1 IHC 28-8 and 22C3 pharmDx assays [66]. The analysis of cytological specimens using the PD-L1 IHC 288 assay may therefore be possible, although the pharmDx kit has been approved only for histological samples [17, 18], and particular care should be taken with fixative types for cytological specimens [67]. Cytological analysis would require reference samples such as cell-line microarrays, which have also shown utility as a control in analysis of tissue microarrays.

The PD-L1 IHC 28-8 pharmDx kit was not developed to assess immune cells. Only one assay, SP142, has been developed to assess immune cell staining in addition to tumor membrane staining [68]. Although interlaboratory concordance within a single assay was acceptable, most studies have found poor concordance between different assays in immune cell staining, compared with tumor membrane staining [58, 69-72]. This current study therefore did not include immune cell staining in the analysis.

The results of the present analysis demonstrate that protocol optimization by individual laboratories should always be guided by comparison with approved assays, in order to prevent false positives and/or false negatives. If the kit or the staining device is not available (the most likely reason for developing an assay within the laboratory), comparison on a set of reference samples with known results, obtained by a reference laboratory using the approved IHC 28-8 pharmDx kit, is considered a prerequisite. Further studies are needed to validate 28-8 assays on sample types used in clinical practice.

\section{Conclusion}

A systematic and comprehensive comparison of PD-L1 immunohistochemistry protocols on the four most commonly used immunohistochemistry platforms using the Abcam anti-PD-L1 28-8 antibody in lung cancer, melanoma, and head and neck cancer, using tissue microarray samples, showed adequate concordance and good reproducibility when compared with the reference PD-L1 IHC 28-8 pharmDx kit. The use of reference samples with known PDL1 expression levels, including cell-line microarrays, helped to confirm the sensitivity and specificity of each of the laboratory-developed assays.

Further investigation is needed to determine protocols for other tumor and biopsy types. All such laboratorydeveloped assays should be validated by direct comparison either with the approved protocol in the $28-8$ pharmDx kit, or with externally validated reference samples if the kit is not available. Each laboratory should perform its own validation prior to use.

Acknowledgments The authors would like to thank the patients who donated samples, as well as the contribution of Alexander Pieper (Bristol-Myers Squibb). Medical writing and editorial support was provided by Eloise Aston, MSc, and Jay Rathi, MA, of Spark Medica Inc (USA), funded by Bristol-Myers Squibb.

\section{Compliance with ethical standards}

Conflict of interest CK, HS, DZ, and BJ are employees of Targos. HUS, SE, and MM-O are employees of Universitätsmedizin Göttingen. CD'A has received honoraria and reimbursements from Roche Pharma, Roche TD, Pfizer and, via Targos, from Bristol-Myers Squibb and MSD. H-US is a consultant for Targos and has received honoraria and reimbursements from Abbott Molecular, Bristol-Myers Squibb, MSD, Novartis, Pfizer, Roche Pharma, ZytoMed, and ZytoVision. JR is a co-founder of Targos and received a fee from Bristol-Myers Squibb for this study.

\section{References}

1. Dyck L, Mills KHG. Immune checkpoints and their inhibition in cancer and infectious diseases. Eur J Immunol. 2017;47:765-79.

2. Pedoeem A, Azoulay-Alfaguter I, Strazza M, et al. Programmed death-1 pathway in cancer and autoimmunity. Clin Immunol. 2014;153:145-52.

3. Ostrand-Rosenberg S, Horn LA, Haile ST. The programmed death-1 immune-suppressive pathway: barrier to antitumor immunity. J Immunol. 2014;193:3835-41.

4. Chen DS, Irving BA, Hodi FS. Molecular pathways: nextgeneration immunotherapy-inhibiting programmed death-ligand 1 and programmed death-1. Clin Cancer Res. 2012;18:6580-7. 
5. Pai SI, Zandberg DP, Strome SE. The role of antagonists of the PD-1:PD-L1/PD-L2 axis in head and neck cancer treatment. Oral Oncol. 2016;61:152-8.

6. Konishi J, Yamazaki K, Azuma M, et al. B7-H1 expression on non-small cell lung cancer cells and its relationship with tumorinfiltrating lymphocytes and their PD-1 expression. Clin Cancer Res. 2004;10:5094-5100.

7. Chen DS, Mellman I. Oncology meets immunology: the cancerimmunity cycle. Immunity. 2013;39:1-10.

8. Barber DL, Wherry EJ, Masopust D, et al. Restoring function in exhausted CD8 T cells during chronic viral infection. Nature. 2006;439:682-7.

9. Pharmaceuticals and medical devices agency. Opdivo [Review report]. 2014. http://www.pmda.go.jp/files/000209430.pdf. Accessed 13 Dec 2016.

10. Bristol-Myers Squibb. OPDIVO [prescribing information]. 2014. http://packageinserts.bms.com/pi/pi_opdivo.pdf. Accessed 8 Dec 2016.

11. Merck \& Co. KEYTRUDA [prescribing information]. 2016. https://www.merck.com/product/usa/pi_circulars/k/keytruda/ keytruda_pi.pdf. Accessed 8 Dec 2016.

12. Genentech, Inc. TECENTRIQ [prescribing information]. 2016. https://www.gene.com/download/pdf/tecentriq_prescribing.pdf. Accessed 23 Jan 2017.

13. Bristol-Myers Squibb. OPDIVO. Summary of product characteristics. 2016. http://www.ema.europa.eu/docs/en_GB/document_ library/EPAR_-_Product_Information/human/003985/WC500189765. pdf. Accessed 23 Feb 2017.

14. Merck \& Co., Inc. KEYTRUDA. Summary of product characteristics. 2017. http://www.ema.europa.eu/docs/en_GB/ document_library/EPAR___Product_Information/human/003820/ WC500190990.pdf. Accessed 20 Feb 2017.

15. F Hoffmann-La Roche AG. TECENTRIQ. Summary of product characteristics. http://www.ema.europa.eu/docs/en_GB/ document_library/EPAR_-_Product_Information/human/004143/ WC500235778.pdf. Accessed 3 Jan 2018.

16. AstraZeneca Pharmaceuticals LP. IMFINZI [prescribing information]. 2017. https://www.azpicentral.com/imfinzi/imfinzi. pdf\#page $=1$. Accessed 3 Jan 2018.

17. Dako. PD-L1 IHC 28-8 pharmDx. 2016. http://www.accessdata. fda.gov/cdrh_docs/pdf15/P150027c.pdf. Accessed 20 Feb 2017.

18. Dako. PD-L1 IHC 28-8 pharmDx (package insert). 2017. http://www.agilent.com/cs/library/packageinsert/public/ P03914EFG_03.pdf. Accessed 10 Aug 2017.

19. Dako. PD-L1 IHC 28-8 pharmDx Interpretation Manual: Urothelial Carcinoma. 2017. http://www.agilent.com/cs/library/ usermanuals/public/29188_pd-11-ihc-28-8-uc-interpretation-ma nual.pdf. Accessed 11 Aug 2017.

20. Mu CY, Huang JA, Chen Y, et al. High expression of PD-L1 in lung cancer may contribute to poor prognosis and tumor cells immune escape through suppressing tumor infiltrating dendritic cells maturation. Med Oncol. 2011;28:682-8.

21. Chen YB, Mu CY, Huang JA. Clinical significance of programmed death-1 ligand-1 expression in patients with non-small cell lung cancer: a 5-year-follow-up study. Tumori. 2012;98:751-5.

22. Sun J, Xu K, Wu C, et al. PD-L1 expression analysis in gastric carcinoma tissue and blocking of tumor-associated PDL1 signaling by two functional monoclonal antibodies. Tissue Antigens. 2007;69:19-27.

23. Sznol M, Chen L. Antagonist antibodies to PD-1 and B7-H1 (PDL1) in the treatment of advanced human cancer. Clin Cancer Res. 2013;19:1021-34.

24. Taube JM, Anders RA, Young GD, et al. Colocalization of inflammatory response with B7-H1 expression in human melanocytic lesions supports an adaptive resistance mechanism of immune escape. Sci Transl Med. 2012;4:127ra137.

25. Novotny J, Cogswell J, Inzunza $\mathrm{H}$, et al. Establishing a complementary diagnostic for anti-PD-1 immune checkpoint inhibitor therapy. Ann Oncol. 2016;27:1966-9.

26. Patel SP, Kurzrock R. PD-L1 expression as a predictive biomarker in cancer immunotherapy. Mol Cancer Ther. 2015;14:847-56.

27. Brahmer J, Reckamp KL, Baas P, et al. Nivolumab versus docetaxel in advanced squamous-cell non-small-cell lung cancer. $\mathrm{N}$ Engl J Med. 2015;373:123-35.

28. Borghaei H, Paz-Ares L, Horn L, et al. Nivolumab versus docetaxel in advanced nonsquamous non-small-cell lung cancer. $\mathrm{N}$ Engl J Med. 2015;373:1627-39.

29. Ng Kee Kwong F, Laggner U, McKinney O, et al. Expression of PD-L1 correlates with pleomorphic morphology and histological patterns of non-small cell lung carcinomas. Histopathology. 2018. https://doi.org/10.1111/his.13466. [Epub ahead of print].

30. Janzic U, Kern I, Janzic A, et al. PD-L1 expression in squamouscell carcinoma and adenocarcinoma of the lung. Radiol Oncol. 2017;51:357-62.

31. Reck M, Rodríguez-Abreu D, Robinson AG, et al. Pembrolizumab versus chemotherapy for PD-L1-positive non-smallcell lung cancer. N Engl J Med. 2016;375:1823-33.

32. Carbone DP, Reck M, Paz-Ares L, et al. First-line nivolumab in stage IV or recurrent non-small-cell lung cancer. N Engl J Med. 2017;376:2415-26.

33. Lee SJ, Jang BC, Lee SW, et al. Interferon regulatory factor-1 is prerequisite to the constitutive expression and IFN-gammainduced upregulation of B7-H1 (CD274). FEBS Lett. 2006;580:755-62.

34. Ribas A. Adaptive immune resistance: how cancer protects from immune attack. Cancer Discov. 2015;5:915-9.

35. Edwards R, Black J, Young T, et al. Characterization of the immunomodulatory effects of nivolumab and ipilimumab in advanced melanoma by quantitative immunohistochemistry. In: 29th European Congress of Pathology: Amsterdam, Netherlands; 2017 (abstract 593).

36. Hato SV, Khong A, de Vries IJ, et al. Molecular pathways: the immunogenic effects of platinum-based chemotherapeutics. Clin Cancer Res. 2014;20:2831-7.

37. Gowrishankar K, Gunatilake D, Gallagher SJ, et al. Inducible but not constitutive expression of PD-L1 in human melanoma cells is dependent on activation of NF-kappaB. PLoS One. 2015;10: $\mathrm{e} 0123410$.

38. Ilie M, Long-Mira E, Bence C, et al. Comparative study of the PD-L1 status between surgically resected specimens and matched biopsies of NSCLC patients reveal major discordances: a potential issue for anti-PD-L1 therapeutic strategies. Ann Oncol. 2016;27:147-53.

39. Mahoney KM, Sun H, Liao X, et al. PD-L1 antibodies to its cytoplasmic domain most clearly delineate cell membranes in immunohistochemical staining of tumor cells. Cancer Immunol Res. 2015;3:1308-15.

40. Dong H, Zhu G, Tamada K, et al. B7-H1, a third member of the B7 family, co-stimulates T-cell proliferation and interleukin-10 secretion. Nat Med. 1999;5:1365-9.

41. Schats K, Vre EAV, Schrijvers D, et al. Epitope mapping of PDL1 primary antibodies (28-8, SP142, SP263, E1L3N). J Clin Oncol. 2017;35:3028.

42. Phillips T, Millett MM, Zhang X, et al. Development of a diagnostic programmed cell death 1-ligand 1 immunohistochemistry assay for nivolumab therapy in melanoma. Appl Immunohistochem Mol Morphol. 2018;26:6-12.

43. Phillips T, Simmons P, Inzunza HD, et al. Development of an automated PD-L1 immunohistochemistry (IHC) assay for 
non-small cell lung cancer. Appl Immunohistochem Mol Morphol. 2015;23:541-9.

44. Alvarez S, Hanks DA, William J, et al. Assay performance of the PD-L1 IHC 28-8 pharmDx assay in squamous cell carcinoma of the head and neck (SCCHN). J Clin Oncol. 2017;35:e14588.

45. De Los Santos R, William J, Hanks DA, et al. Development of a diagnostic PD-L1 immunohistochemical assay for nivolumab therapy in urothelial carcinoma. J Clin Oncol. 2017;35:e14585.

46. Adam J, Le Stang N, Rouquette I, et al. Multicenter French harmonization study for PD-L1 IHC testing in non-small cell lung cancer. Ann Oncol. 2018;29:953-8.

47. Abcam. Anti-PD-L1 antibody [28-8] (ab205921). 2017. http://www.abcam.com/pd-11-antibody-28-8-ab205921.html. Accessed 13 Jan 2017.

48. Dako. Autostainer Link 48 immunohistochemistry staining system. http://www.dssimage.com/images/upload/AutostainerLink48. pdf. Accessed $24 \mathrm{Feb} 2017$.

49. Dako Omnis. PD-L1 staining protocol with anti-PD-L1 clone 28-8 (Abcam) on Dako Omnis. 2015. http://www.agilent.com/cs/libra ry/datasheets/public/29093_dako-omnis-us-data-sheet.pdf. Accessed 14 Aug 2017.

50. Leica Biosystems. Leica Bond III. Fully automated IHC \& ISH. 2012. https://drp8p5tqcb2p5.cloudfront.net/fileadmin/downloads_ lbs/Leica\%20BOND-ADVANCE/Brochures/BOND-III_ Brochure_us.pdf. Accessed 24 Feb 2017.

51. Ventana Medical Systems, Inc. BenchMark Ultra slide staining system specifications. 2013. http://www.ventana.com/documents/ BMK_ULTRA_Spec_Sheet_web.pdf. Accessed 24 Feb 2017.

52. Horizon Discovery Group. CD274 (PD-L1) Expression IHC Reference Standard. 2017. https://www.horizondiscovery.com/ cd274-pd-11-expression-ihc-reference-standard-hd787. Accessed 1 Mar 2017.

53. Batenchuk C, Albitar M, Sudarsanam S, et al. A comparative study of PD-L1 IHC 22C3 and 28-8 FDA-approved diagnostic assays in cancer. AACR: Washington, D.C.; 2017. (Abstract 4015).

54. Neuman T, London M, Kania-Almog J, et al. A harmonization study for the use of 22C3 PD-L1 immunohistochemical staining on Ventana's platform. J Thorac Oncol. 2016;11:1863-8.

55. Cogswell J, Inzunza $\mathrm{H}$, Wu Q, et al. An analytical comparison of Dako 28-8 pharmDx assay and an E1L3N laboratory-developed test in the immunohistochemical detection of programmed deathligand 1. Mol Diagn Ther. 2017;21:85-93.

56. Smith J, Robida M, Acosta K, et al. Quantitative and qualitative characterization of two PD-L1 clones: SP263 and E1L3N. Diagn Pathol. 2016;11:44.

57. Ilie M, Khambata-Ford S, Copie-Bergman C, et al. Use of the $22 \mathrm{C} 3$ anti-PD-L1 antibody to determine PD-L1 expression in multiple automated immunohistochemistry platforms. PLoS One. 2017;12:e0183023.

58. Buttner R, Gosney JR, Skov BG, et al. Programmed death-ligand 1 immunohistochemistry testing: a review of analytical assays and clinical implementation in non-small-cell lung cancer. J Clin Oncol. 2017;35:3867-76.

59. Marchetti A, Barberis M, Franco R, et al. Multicenter comparison of 22C3 pharmDx (Agilent) and SP263 (Ventana) assays to test PD-L1 expression for NSCLC patients to be treated with immune checkpoint inhibitors. J Thorac Oncol. 2017;12:1654-63.

60. Cree I, Booton R, Cane P, et al. PD-L1 testing for lung cancer in the UK: recognizing the challenges for implementation. Histopathology. 2016;69:177-86.

61. Brunnstrom H, Johansson A, Westbom-Fremer S, et al. PD-L1 immunohistochemistry in clinical diagnostics of lung cancer: inter-pathologist variability is higher than assay variability. Mod Pathol. 2017;30:1411-21.

62. Gniadek TJ, Li QK, Tully E, et al. Heterogeneous expression of PD-L1 in pulmonary squamous cell carcinoma and adenocarcinoma: implications for assessment by small biopsy. Mod Pathol. 2017;30:530-8.

63. Gaule P, Smithy JW, Toki M, et al. A quantitative comparison of antibodies to programmed cell death 1 ligand 1. JAMA Oncol. 2017;3:256-9.

64. Rehman JA, Han G, Carvajal-Hausdorf DE, et al. Quantitative and pathologist-read comparison of the heterogeneity of programmed death-ligand 1 (PD-L1) expression in non-small cell lung cancer. Mod Pathol. 2017;30:340-9.

65. Dix Junqueira Pinto G, de Souza Viana L, Scapulatempo Neto C, et al. Evaluation of PD-L1 expression in tumor tissue of patients with lung carcinoma and correlation with clinical and demographic data. J Immunol Res. 2016;2016:9839685.

66. Skov B, Skov T. Paired comparison of PD-L1 expression on cytologic and histologic specimens from malignancies in the lung assessed with PD-L1 IHC 28-8 pharmDx and PD-L1 IHC 22C3 pharmDx. Appl Immunohistochem Mol Morphol. 2017;25:453-9.

67. van Essen HF, Verdaasdonk MA, Elshof SM, et al. Alcohol based tissue fixation as an alternative for formaldehyde: influence on immunohistochemistry. J Clin Pathol. 2010;63:1090-4.

68. Ventana Medical Systems, Inc. VENTANA PD-L1 (SP142) Assay [instructions for use]. 2016. http://www.accessdata.fda.gov/ cdrh_docs/pdf16/P160002c.pdf. Accessed 20 Feb 2017.

69. Hirsch FR, McElhinny A, Stanforth D, et al. PD-L1 immunohistochemistry assays for lung cancer: results from phase 1 of the Blueprint PD-L1 IHC Assay Comparison Project. J Thorac Oncol. 2017;12:208-22.

70. Scheel AH, Dietel M, Heukamp LC, et al. Harmonized PD-L1 immunohistochemistry for pulmonary squamous-cell and adenocarcinomas. Mod Pathol. 2016;29:1165-72.

71. Scheel AH, Baenfer G, Baretton G, et al. Interlaboratory concordance of PD-L1 immunohistochemistry for non-small-cell lung cancer. Histopathology. 2018;72:449-59.

72. Rimm DL, Han G, Taube JM, et al. A prospective, multi-institutional, pathologist-based assessment of 4 immunohistochemistry assays for PD-L1 expression in non-small cell lung cancer. JAMA Oncol. 2017;3:1051-8. 